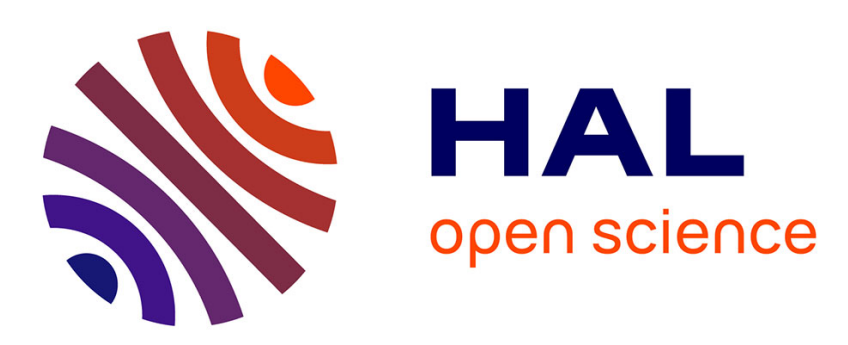

\title{
Human-Robot-Interfaces based on Mixed Reality for Underwater Robot Teleoperation
}

\author{
Christophe Domingues, Mouna Essabbah, Nader Cheaib, Samir Otmane,
} Alain Dinis

\section{- To cite this version:}

Christophe Domingues, Mouna Essabbah, Nader Cheaib, Samir Otmane, Alain Dinis. Human-RobotInterfaces based on Mixed Reality for Underwater Robot Teleoperation. 9th IFAC Conference on Manoeuvring and Control of Marine Craft (MCMC 2012), Sep 2012, Arenzano, Italy. pp.212-215, 10.3182/20120919-3-IT-2046.00036 . hal-00761894

\section{HAL Id: hal-00761894 \\ https://hal.science/hal-00761894}

Submitted on 12 Dec 2012

HAL is a multi-disciplinary open access archive for the deposit and dissemination of scientific research documents, whether they are published or not. The documents may come from teaching and research institutions in France or abroad, or from public or private research centers.
L'archive ouverte pluridisciplinaire HAL, est destinée au dépôt et à la diffusion de documents scientifiques de niveau recherche, publiés ou non, émanant des établissements d'enseignement et de recherche français ou étrangers, des laboratoires publics ou privés. 


\title{
Human-Robot-Interfaces based on Mixed Reality for Underwater Robot Teleoperation
}

\author{
Christophe Domingues*, Mouna Essabbah*, Nader Cheaib* and Samir Otmane* \\ Alain Dinis** \\ *University of Evry - IBISC EA 4526, France \\ (e-mail: surname.name@ibisc.univ-evry.fr). \\ **VirtualDive SAS, Versailles, France (e-mail: \\ adinis@virtualdive.com)
}

\begin{abstract}
In this paper, we present two Human-Robot interfaces for underwater robot teleoperation. This work is in the context of the Digital Ocean Europe project ${ }^{1}$ that aims at digitalizing seafloor sites in 3D imagery using underwater robots (ROVs), and uses this information in order to edit interactive, virtually animated environments that are diffused online. The work presented in this paper concerns the 1) Web interface installed on an aquatic PC called DOLPHYN and 2) a VR/AR semi-immersive platform. Both interfaces are used for remote exploration of underwater sites. We discuss their technical implementation for underwater robot teleoperation (ROV).
\end{abstract}

Keywords: Teleoperation, ROV, Underwater, Distributed Architecture, Virtual Reality, Augmented Reality, Assistance to 3D interaction.

\section{INTRODUCTION}

Putting in practice the domains of Virtual and Augmented Reality (VR/AR) in aquatic leisure activities constitutes a technological rupture when compared with the status of related technologies. In fact, with the extension of Internet to underwater applications, the innovative character of the Digital Ocean project that we introduce in this paper becomes evident, and the technological impact of this development in the littoral and beach tourism is considerable. In consequence, a new form of durable tourism will surge and beaches will evolve to become playgrounds for entertainment, knowledge and social intercourse.

Our goal is to develop collaborative methods of virtual diving for the teleoperation of a remote operated vehicle (ROV) placed in a given diving site. In fact, the ROV is equipped with video cameras and artificial lights, along with other instruments (compass, depth) ([Roston et al., 2007] and [Jenkyns et al., 2010]).

In this paper, we present two types of Human-RobotInterfaces (HRI) that are developed for underwater robot teleoperation. The first one is a Web interface, enabling users, independently of their location, to control and teleoperate the ROV. In the Digital Ocean project, we have implemented a web interface on a special aquatic computer called DOLPHYN that simulates scuba diving [Domingues et al., 2012]. In fact, there are recent developments in order to extend the use of computers and computer components, such as the mouse, to underwater usage ([Dinis et al., 2008] and [Ashok et al., 2003]). However, in the literature, there is no work of an underwater-computerized display system such as the DOLPHYN.
The second interface we present in this paper for underwater robot teleoperation is based on VR/AR, which gives the user a multisensory exploration of the underwater site. This fact enhances the feeling of presence due to stereoscopic display, $3 \mathrm{D}$ interaction, as well as a haptic interface in order to allow task execution involving human beings and robots at the same workspace, which is also called telepresence. Similar work can be cited in this context such as the work in [Otmane et al., 2000] or [Greena et al., 2008]. In our system, the two HRI can be used simultaneously for collaborative diving scenarios. Both HRI are developed in the context of the Digital Ocean Europe project, and can be seen in Figure 1.

\section{TELEOPERATION SPECIFICATIONS}

As mentioned, the first HRI is the DOLPHYN, which diffuses multimedia content for scuba divers. This submersible device integrates a x86 tablet-PC running on Windows 7. In fact, the DOLPHYN, connected to the internet, provides to the scuba diver two integrated joysticks in order to teleoperate the ROV. Concerning the second HRI, it is based on a VR/AR platform giving users a multisensory exploration of the underwater site while enhancing the feeling of presence using a stereoscopic display and a haptic interface. 


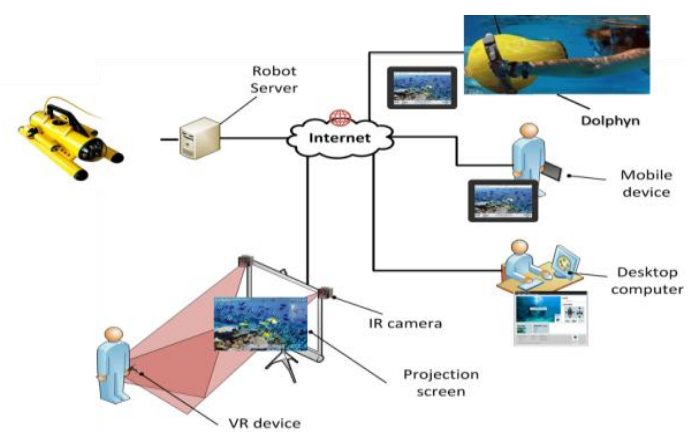

Fig. 1: Users can collaborate together using different HRI to teleoperate the ROV.

In fact, four types of applications are used in our system: Client side application (Web/DOLPHYN or VR) representing the diving, the multiuser service, the ROV application and the video streaming service allowing to control the distant ROV and capture video images. The user interacts with the application in order to visualize the ROV camera, where he/she can also move or rotate the distant ROV in order to modify his/her point of view using the Internet network. This is possible through some design constraint that we took in consideration:

- Server applications do not know the state of the main application, so they act as a slave.

- Orders and commands can solely be issued from the Client side applications, where the ROV application is only responding to those orders;

- The infrastructure linking the three applications uses the internet network through TCP/IP;

- The connection must be bilateral in order to guarantee the data transfer. The ROV is connected to its server using a RJ45 connection, which in turn is connected to Internet via WIFI or $3 \mathrm{G}$.

Indeed, the real-time streaming service allows bringing the media and broadcast to the Internet. The process involves a camera on the ROV, an encoder to digitize the content as well as a content delivery network in order to distribute and deliver the content. The media can then be viewed by endusers in real time. Grabbing, encoding and delivering the stream will be managed by VideoLAN. For encoding the PAL signal, we have chosen the Ogg format, where we use the HTTP protocol for delivery. Secondly, the multiuser service is used to synchronize data between users performing a collaborative virtual diving. Finally, the ROV application is used to accept network connections and transfer commands sent by users to ROV. It also used to prevent multiple and heavy connections to the ROV through the use of a priority list.

\section{HUMAN ROBOT INTERFACES}

\subsection{Web and DOLPHYN Human Robot Interfaces}

The DOLPHYN (see Figures 2 and 3) allows recreating the sensorial conditions of scuba diving in public most frequented aquatic spaces - beaches and swimming pools. The DOLPHYN is autonomous, mobile, easily transported by one person in a backpack, quickly installed and equipped with GPS and wireless systems. It uses an x86 tablet connecting various devices: user interface device, GPS, sensors (accelerometer, thermometer, flow-meter) and a WIFI device. In fact, three modules compose the prototype:

- Waterproof case containing the tablet;

- Waterproof electronic case used to manage all connected devices;

- Body and joysticks.

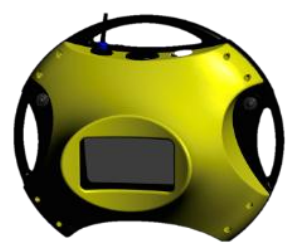

Fig. 2: The 3D Model of the DOLPHYN

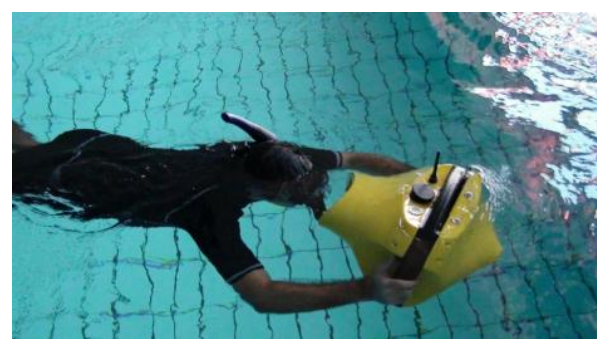

Fig. 3: DOLPHYN testing in a swimming pool

\subsubsection{Web and DOLPHYN Human Robot Interface}

DOLPHYN can remotely teleoperate the ROV via Internet using a simple WEB browser (see Figure 4). In order to control the ROV, the flow meter ( $\mathrm{z}$ axis) is used to send a forward command, and a left joystick is used to send a left/right/up/down commands. DOLPHYN front camera enables the user to see his/her real trajectory in the swimming pool or the sea.

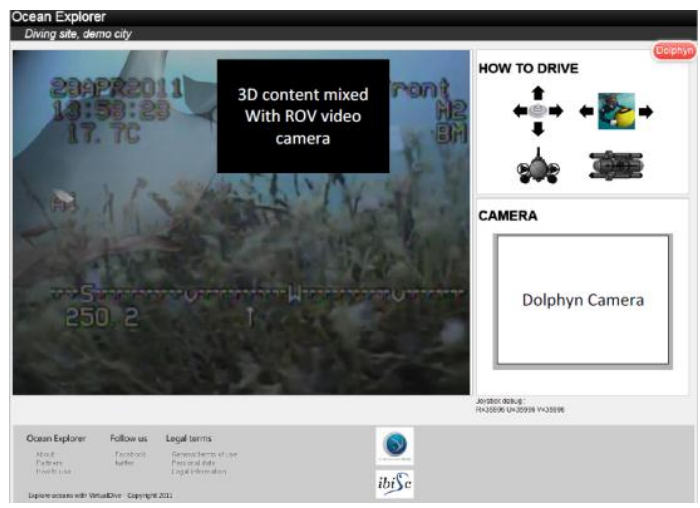

Fig. 4: Web HRI for the DOLPHYN

\subsubsection{The DOLPHYN: Aquatic computer}


The Web HRI for mobile or desktop computers (see Figure 5) allows to send commands to the ROV (2) and supervise sensors data from $\operatorname{ROV}(1,3$ and 5). It also enables a user to use various functionalities (chat or Web services) in the Web interface (part 4 and 6 in Figure 5).

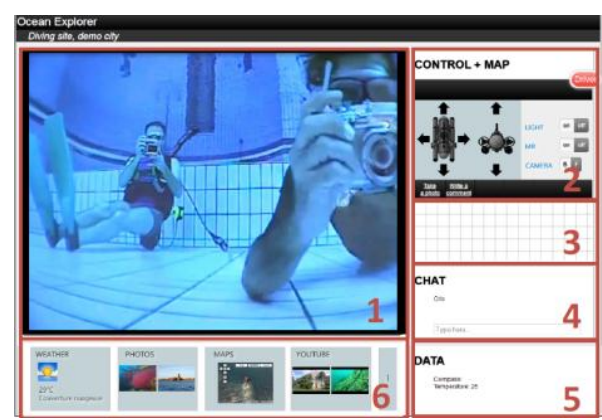

Fig. 5: Web HRI for mobile devices or desktop computers.

Those HRI can also use Augmented Reality technologies by the reorganization of $2 \mathrm{D}$ real markers (see Figure 6). Those markers can be used to add multimedia data (text, images, videos, fauna and flora $3 \mathrm{D}$ models, etc.) or to localize the ROV using its camera.

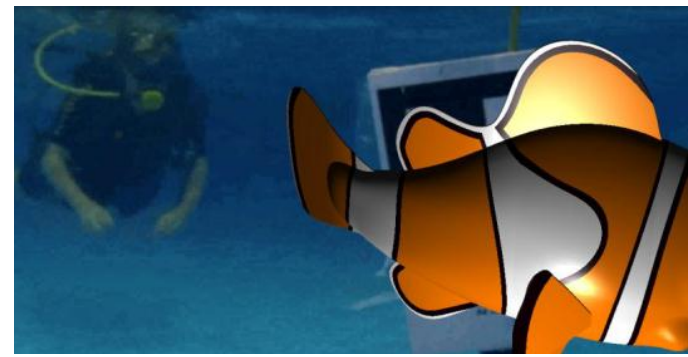

Fig. 6: Use of Augmented Reality to display 3D model

\subsection{Semi-immersive Human Robot Interface}

In order to effectively tele-operate a remote robot, the human-robot interface must provide tools to perceive the remote environment, to make decisions, and to generate commands. We cite similar work such as [Santamaria 2002], [Hamzah 2008] or [Lin 2001], which introduce a ROV safety domain. Furthermore, we attempt to maximize information transfer while minimizing cognitive and sensorimotor workload. We used multimodal interface that contains stereoscopic viewing and haptic feedback to have a more intuitive human-robot interaction. In fact, it aims to reduce training and to overcome the unfamiliarity with VR systems. This interface should improve the feeling of presence and awareness among users doing a virtual seabed exploration, while providing two types of diving:

1) A simulated dive in a virtual environment without real control of the ROV. It also allows users to learn and test a path before the real exploration (via the ROV).

2) A dive in the Mixed Reality (MR) environment via ROV teleoperation.
The used human scale semi-immersive platform is composed of a large screen $(3.2 \mathrm{mX} 2.4 \mathrm{~m})$ and a DLP projector $120 \mathrm{~Hz}$ MIRAG E4000 that provides active stereoscopy. We use stereoscopic glasses Crystal Eyes 3 and their corresponding transmitter. The interaction is provided by 6 degrees of freedom force feedback device named SPIDAR (SPace Interface Device for Artificial Reality) ((1) in Figure 7). The control system is ensured by a handheld flystick ((2) in Figure 7) that includes a set of markers for real time tracking, using two infrared cameras ARTTrack1 ((3) in Figure 7). The platform is illustrated in Figure 7. Communication between the VR/AR platform and the ROV is made via the Internet as well as the human robot interface described in section 3.1.

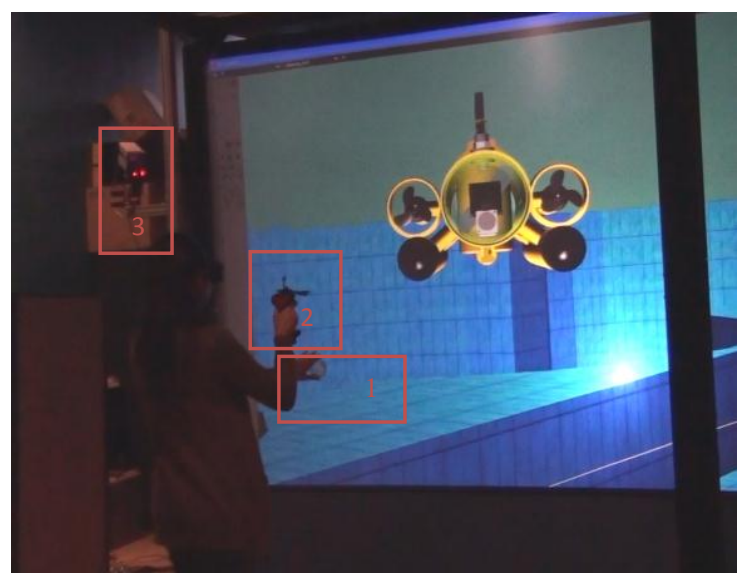

Fig. 7: Semi immersive VR/AR platform

\subsubsection{VR/AR teleoperation interface}

The virtual environment consists of the simulating seabed dive site. The navigation task in the virtual scene is the ROV teleoperation. Thus we created a virtual ROV (see Figure 8) to simulate movements of the real ROV. The navigation using the virtual ROV is done according to the marker placed on the SPIDAR effector, which reproduces position movements of the user. By manipulating the virtual ROV, the operator is controlling the real ROV. The system control allows to manage the robot features (camera switch, activate lights) or show instructions for effective usage.

The visual modality is used to display this main information: 1) Live video stream acquired by the camera on the ROV, how to control camera and switch from one to the other; 2) virtual and interactive $3 \mathrm{D}$ environment representing the explored site; 3) a top view 2D map of the explored site. Complementary information (data from sensors) is also displayed. The force feedback can simulate collisions and increase the feeling of navigation in water (viscosity, marine current, etc.). Audible information may complete the data given by the virtual diving interface to inform diver of events such as the thrusters' sound (which indicates any troubleshooting issues, as well as the ROV's speed).

\subsubsection{VR/AR semi-immersive platform}



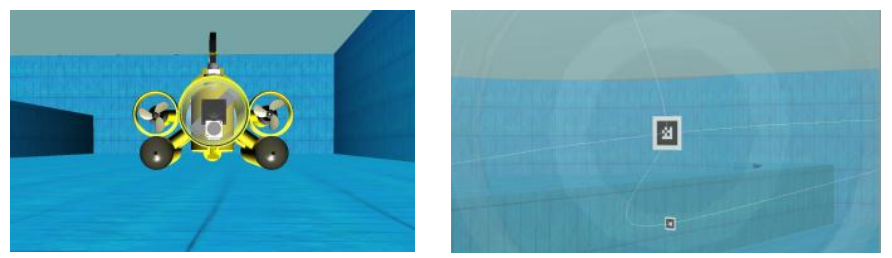

Fig. 8: View from a third camera and embedded camera of the ROV in the test environment (swimming pool)

This multimodality is also used in the 3D interaction assistance. Indeed, assembling the different problems due to human, environment and teleoperation factors, we observed some technical constraints (Loss of ROV maneuverability, transmission delay/stop, etc.) affecting the application's usage (navigation precision, safety of the robot and spatial awareness for diver). Therefore, we applied an assistance model [Essabbah 2010] that includes a set of guides (virtual fixtures) in order to enforce the application's constraints. To give an example of a virtual fixture, the operator must choose a diving path that he/she must then follow. In this case, to be as accurate as possible, we show a $3 \mathrm{D}$ curve representing this path as well as an arrow directed towards the trajectory.

To assist the user, we used the switch between real and virtual navigation. We also changed system's autonomy through the correspondence between the real and virtual ROV. The user can choose to control the real ROV through the ROV embedded video camera. In this case, we calculate the corresponding trajectory of the virtual ROV that is not visualized. The user can also control the virtual ROV through the VR interface. In this case, the real ROV moves as well. Coherence between the real ROV position and the virtual one is provided by real 2D markers (used in Section 3.1.2). When these markers are seen by the real ROV camera, an estimation of the camera position is achieved. Once the position (and even orientation) of the ROV camera is calculated according to the 2D markers positions, we calculate the position of the real ROV in its environment. Positions of the 2D markers being known, we use them as waypoints on the diving path.

\section{CONCLUSION}

In this paper, we have proposed a virtual diving system through the teleoperation of a ROV. The specifications of this teleoperation based on the Internet enable to remotely control the ROV via various human robot interfaces (mobile device, desktop computer, etc.). Firstly, we presented an original interface, the DOLPHYN, which is an aquatic computer. This user-friendly and intuitive interface allows the operator to control the ROV while having the feel of a real dive due to immersion in water. The second human robot interface is based on multimodality, provided by a semi-immersive platform in order to simulate the diving sensation. The methods used allow intuitive interaction and increase the sense of presence of users. In both cases, real 2D markers placed in the water allow to display multimedia content as well as to locate the ROV.
However, a direct link between these two interfaces in order to optimize communication, coordination and cooperation between users teleoperating the robot, is still missing due to interoperability constraints. Hence, one of our objectives is to design a groupware (collaborative software) architecture that specifically addresses interoperability concerns between system's components. One solution is the use of a serviceoriented architecture and Web services in order to provide an XML link between the various interfaces used to teleoperate the robot. This fact enables the exchange of common services between these interfaces in order to optimize the collaborative and social experience among individuals teleoperating the robot.

\section{REFERENCES}

Ashok R. L. and Agrawal D. P. (2003), Next-Generation Wearable Networks. In IEEE Computer 36, 31-39.

Dinis A., Fies N., Cheaib N., Otmane S., Mallem M., Nisan A., Boi J.M.(2008), DIGITAL OCEAN: A National Project for the creation and distribution of Multimedia Content for Underwater Sites. In Proc. Of the 14th International Conference on Virtual Systems and MultiMedia, Dedicated to Digital Heritage, VSMM'08, pages 389- 396, Limassol Cyprus.

Domingues C., Otmane S., Dinis A. (2012), A new Device for Virtual or Augmented Underwater Diving. In IEEE Symposium on 3D User Interface (3DUI 2012).

Essabbah M., Otmane S., Hérisson J. and Mallem M., A New Approach to Design an Interactive System for Molecular Analysis, Lecture Notes in Computer Science (LNCS 5613), Human-Computer Interaction, (HCII 2009), pp 713-722, Springer-Verlag Berlin Heidelberg, 2009.

Greena S. A., Billinghurstb M., Qi Chena X. and Chase J. G. (2008). Human-Robot Collaboration: A Literature Review and Augmented Reality Approach in Design.

Hamzah M. S. M., Zakaria M., Abd Jalil M. F. I., and Zamli K. Z.. 3D virtual simulation software for underwater application. In 2nd International Conference Underwater System Technology, 2008.

Jenkyns R. (2010), "NEPTUNE Canada: Data integrity from the seafloor to your (Virtual) Door". In IEEE OCEANS 2010, p. 1-7.

Lin Qingping and Kuo Chengi. On applying virtual reality to underwater robot tele-operation and pilot training. The International Journal of Virtual Reality, 5(1), 2001.

Otmane S., Mallem M., Kheddar A., and Chavand F. (2000), Ariti: an augmented reality interface for teleopeation on the Internet. In Advanced Simulation Technologies Conf. (ASTC2000), pages 254-261. Wyndham City Center Hotel, Washington, D.C. (USA).

Roston J., Bradley C., Cooperstock JR. (2007), Underwater window: high definition video on VENUS and NEPTUNE. In IEEE OCEANS 2007, p. 1-8.

Santamaria Juan C., Opdenbosch Augusto, XYZ Solutions, Inc. Monitoring Underwater Operations with Virtual Environments. In Offshore Technology Conference, 2002. 